\title{
Sarcoidlike Reaction of Neoplasia Causing Hypermetabolic Thoracic Adenopathy in Setting of Extrathoracic Malignancy: Report of Two Cases and a Review of the Differential Diagnostic Considerations
}

\author{
Jonathan B. Craun ${ }^{1}$, Kevin P. Banks ${ }^{1-3}$, Michael N. Clemenshaw ${ }^{1,2}$, and Ronald W. Moren ${ }^{1}$ \\ ${ }^{1}$ Department of Radiology, Brooke Army Medical Center, Fort Sam Houston, Houston, Texas; ${ }^{2}$ Department of Radiology, University of \\ Texas Health Science Center at San Antonio, San Antonio, Texas; and ${ }^{3}$ Rays, Littleton, Colorado
}

\begin{abstract}
The development of noncaseating granulomas in a patient with underlying malignancy and no symptoms or signs suggestive of systemic sarcoid is often referred to as a sarcoidlike reaction and is estimated to occur in a small but significant portion of cancer patients. The pathogenesis is poorly understood, but the entity is hypothesized to be an immune phenomenon representing a host defense mechanism against the spread of tumor cells. Sarcoidlike reactions can occur at any time from the time of diagnosis to several years afterward and may occur in lymph nodes draining a malignant tumor, in the tumor itself, and even in nonregional tissues. Like sarcoid, sarcoidlike reactions of neoplasia can demonstrate hypermetabolic lymph nodes on ${ }^{18} \mathrm{~F}-\mathrm{FDG}$ PET imaging and thus be readily confused with metastatic disease. We describe 2 cases of a sarcoidlike reaction of neoplasia presenting as hypermetabolic thoracic lymph nodes on ${ }^{18} \mathrm{~F}$-FDG PET/CT obtained for follow-up of extrathoracic malignancies: one a 73-y-old woman with a history of stage III head and neck squamous cell carcinoma and the other a 34-yold woman with recurrent giant cell tumor of the sacrum. In both instances, the differential diagnosis for the finding of hypermetabolic thoracic lymph nodes included the possibility of a sarcoidlike reaction, though tissue sampling was pursued to exclude the more worrisome presence of metastatic disease or, less likely, a new primary malignancy. We review the topic of sarcoidlike reactions of neoplasia as well as the analytic approach to hypermetabolic mediastinal and hilar lymph nodes encountered on ${ }^{18} \mathrm{~F}-\mathrm{FDG}$ PET/CT.
\end{abstract}

Key Words: sarcoidlike reaction; ${ }^{18} \mathrm{~F}-\mathrm{FDG}$ PET/CT; hypermetabolic lymph nodes; lymph node metastases

J Nucl Med Technol 2012; 40:231-235

DOI: $10.2967 /$ jnmt.112.102814

A sarcoidlike reaction of neoplasia can demonstrate hypermetabolic lymph nodes on ${ }^{18}$ F-FDG PET imaging

\footnotetext{
Received Jan. 11, 2012; revision accepted Jun. 1, 2012.

For correspondence contact: Kevin Banks, 3851 Roger Brooke Dr., Fort Sam Houston, TX 78234.

E-mail: kevin.banks@us.army.mil

Published online Sep. 26, 2012.

COPYRIGHT @ 2012 by the Society of Nuclear Medicine and Molecular Imaging, Inc.
}

and thus be readily confused with metastatic disease. Here, we describe 2 cases of a sarcoidlike reaction of neoplasia presenting as hypermetabolic thoracic lymph nodes on ${ }^{18} \mathrm{~F}$ FDG PET/CT obtained for follow-up of extrathoracic malignancies.

\section{CASE REPORT}

Patient A was a 73-y-old woman with a history of squamous cell carcinoma of the larynx who presented for follow-up ${ }^{18} \mathrm{~F}$-FDG PET/CT 3 mo after completing chemoradiation. Before therapy, the patient had been diagnosed with stage III disease without evidence of local, regional, or distal spread (T3M0N0) (Fig. 1). Clinical examination just before follow-up imaging was negative for any evidence of active disease. ${ }^{18}$ F-FDG PET/CT showed no soft-tissue lesion or abnormal metabolic activity in the region of the prior tumor, though new hypermetabolic lymph nodes were noted in the mediastinum and hila, both of which were enlarged and subcentimeter in size (Fig. 2). No other concerning anatomic or metabolic findings were present. Review of the patient's medical record was negative for any history of sarcoid, and both the patient and her referring provider confirmed the lack of any pulmonary or systemic evidence to suggest occult sarcoid or active infection. Although the findings were most concerning for metastatic disease, the patient's prior staging and benign clinical examination made this less likely and the possibility of a sarcoidlike reaction was postulated. The patient went on to undergo bronchoscopy and biopsy of the subcarinal hypermetabolic lymph node. Histologic analysis demonstrated noncaseating epithelioid granulomas without evidence of malignancy. The subsequent one and a half years of clinical and imaging follow-up were negative for evidence of recurrent or metastatic disease or of sarcoid. The hypermetabolic mediastinal and hilar lymph nodes remained unchanged on 2 subsequent ${ }^{18} \mathrm{~F}$-FDG PET/CT examinations.

Patient B was a 34-y-old woman with a history of giant cell tumor of the sacrum diagnosed almost $4 \mathrm{y}$ before the $\mathrm{PET} / \mathrm{CT}$ examination. The original tumor had arisen at S2 and extended into both S1 and S3 and was treated with 


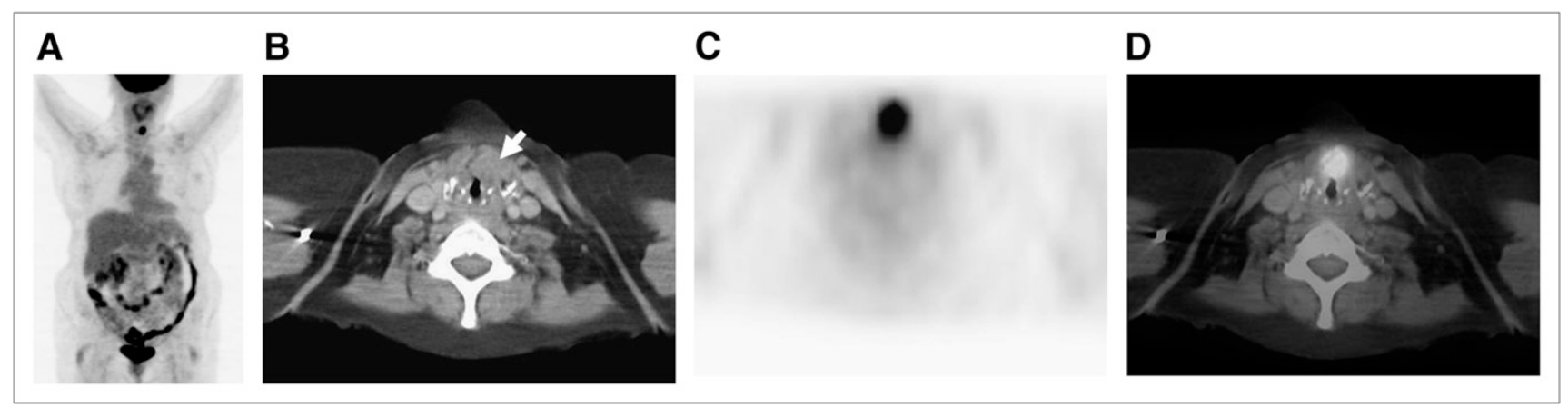

FIGURE 1. ${ }^{18} \mathrm{~F}-\mathrm{FDG}$ PET/CT for initial staging of patient $A$ 's laryngeal cancer. (A) Maximum-intensity projection demonstrates single focus of hypermetabolic activity at location of laryngeal cancer with no evidence of locoregional spread of distal metastases. (B) CT shows abnormal soft-tissue mass (arrow) along anterior aspect of left vocal cord. (C and D) Axial attenuation-corrected PET and PET/CT, respectively, demonstrate that lesion is hypermetabolic, consistent with known diagnosis of squamous cell carcinoma.

partial sacrectomy. Less than 1 y later, the patient experienced local recurrence, which was again treated with surgery and subsequent radiation therapy. Three years later, she underwent PET/CT for suspected recurrence. The examination demonstrated a hypermetabolic lesion at the site of prior disease, as well as hypermetabolic mediastinal, hilar, right axillary, and right neck lymph nodes, both enlarged and subcentimeter in size (Fig. 3). The patient underwent biopsy of the neck lymph nodes, which, as in patient A, revealed noncaseating epithelioid granulomas without evidence of malignancy. Both patient and provider confirmed the lack of any pulmonary or systemic evidence to suggest sarcoid or infection. Six months later, because of stable findings of hypermetabolic adenopathy, biopsy of the right hilar subcarinal lymph nodes was performed and showed noncaseating epithelioid granulomas without evidence of malignancy, findings identical to those of the prior neck lymph node biopsy. An interval follow-up ${ }^{18}$ F-FDG PET/CT examination 6 mo after the last biopsy demonstrated stable hypermetabolic adenopathy with no evidence of local recurrent disease or new abnormality.

\section{DISCUSSION}

Increased ${ }^{18} \mathrm{~F}$-FDG uptake in lymph nodes is often encountered in patients undergoing PET. In a patient with a history of extrathoracic malignancy, such a finding within hilar and mediastinal lymph nodes is commonly secondary to metastatic disease. However, it is important to keep in mind the many diseases that may cause false-positive findings and to consider an alternative diagnosis in appropriate cases to avoid an erroneous interpretation of metastatic disease. The differential diagnosis in such patients who present with new hypermetabolic hilar and mediastinal adenopathy is extensive and, in addition to metastases, includes lung cancer with nodal spread of disease, lymphoma, infection (to include tuberculosis), sarcoid, other granulomatous diseases such as silicosis or asbestosis, and a sarcoidlike reaction to malignancy.

The predictive accuracy and the false-positive and falsenegative results of ${ }^{18} \mathrm{~F}$-FDG PET in detecting metastatic mediastinal lymph node involvement are $83 \%, 13 \%$, and $9 \%$, respectively (1). Clinical history, laboratory values, and imaging features can help narrow the differential diagnosis, although tissue sampling or biopsy is often necessary to make a definitive diagnosis.

In these 2 cases, the hypermetabolic hilar and mediastinal lymph nodes were the result of a sarcoidlike reaction. The development of noncaseating granulomas in a patient with underlying malignancy and no symptoms or signs suggestive of systemic sarcoid is often referred to as a

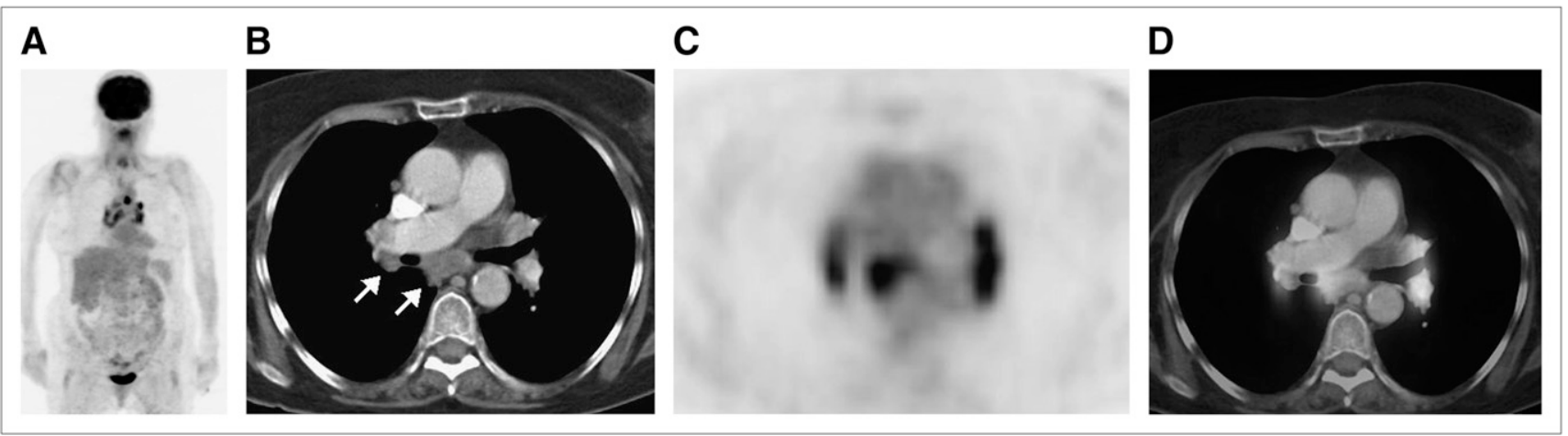

FIGURE 2. Follow-up ${ }^{18}$ F-FDG PET/CT for routine surveillance of patient A's laryngeal cancer. Clinically, patient had no evidence of disease. (A) Maximum-intensity projection demonstrates bilateral abnormal ${ }^{18} \mathrm{~F}-\mathrm{FDG}$ avidity in hilar regions and mediastinum. (B-D) Axial CT, PET, and PET/CT through hila and mediastinum show these foci to correspond to hypermetabolic lymph nodes (arrows). 


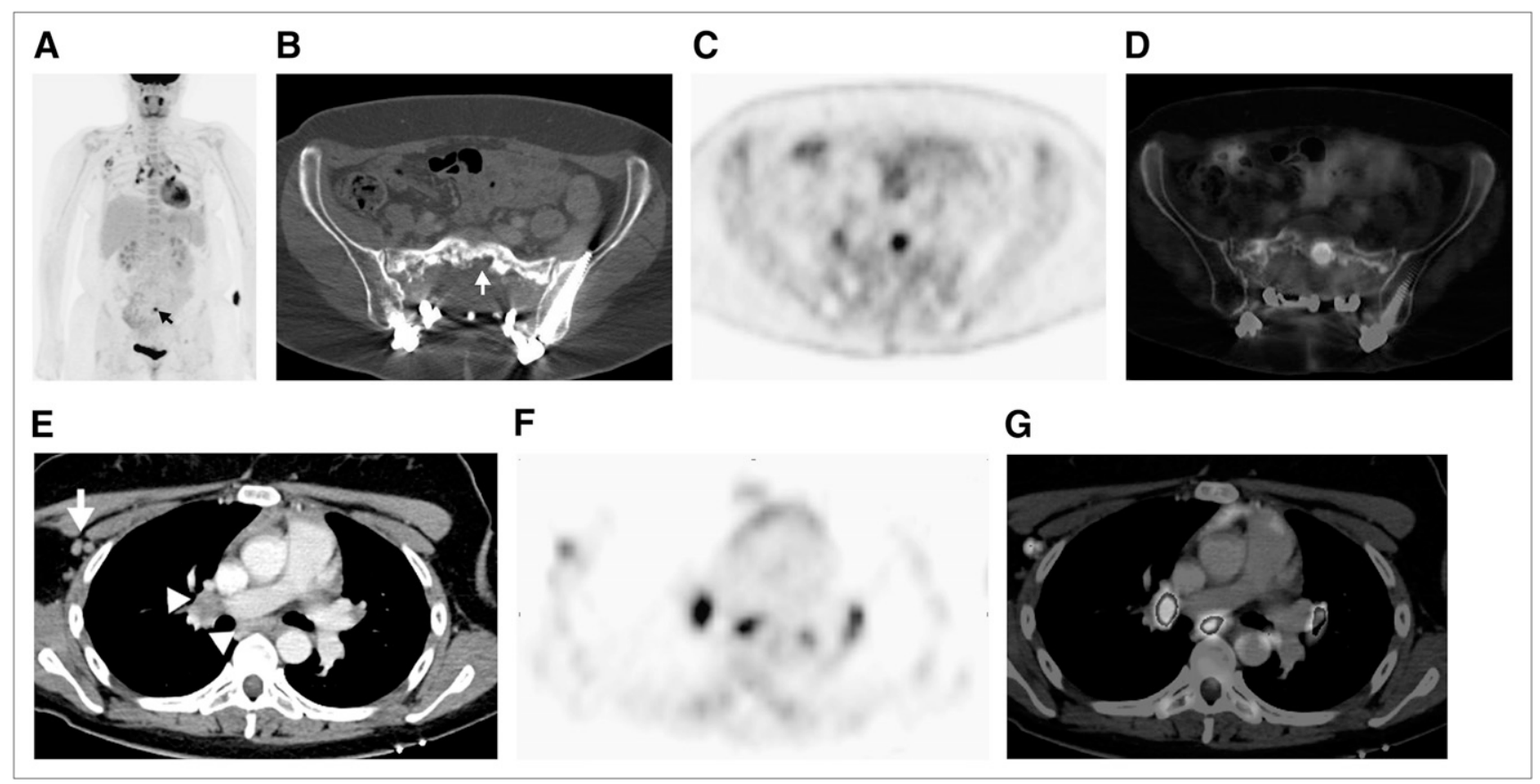

FIGURE 3. (A) Maximum-intensity projection from follow-up ${ }^{18} \mathrm{~F}-\mathrm{FDG}$ PET/CT done for routine surveillance of patient B demonstrates abnormal ${ }^{18} \mathrm{~F}-\mathrm{FDG}$ avidity in sacrum at site of prior disease (arrow). (B, C, and D) Axial CT, PET, and PET/CT, respectively, show that sacral activity corresponds to new hypermetabolic osteolysis consistent with local recurrence. The combination was believed to represent local recurrent disease. Additional findings on maximum-intensity projection included new, bilateral hypermetabolic foci in hilar regions and mediastinum, as well as in right axilla and right neck base. (E, F, and G) Axial CT, PET, and PET/ $\mathrm{CT}$, respectively, show these foci to represent hypermetabolic hilar and mediastinal (arrowheads) and right axillary (arrow) lymph nodes. Foci of uptake in right neck base were also shown to be hypermetabolic lymph nodes.

sarcoidlike reaction (2). It is especially important to consider sarcoidlike reactions in this group of patients, as there is evidence to suggest a relationship between a sarcoid reaction and patients with underlying cancer (2). It is estimated that $4 \%-14 \%$ of all patients with cancer exhibit histopathologic evidence of a sarcoidlike reaction, and this has been described in association with many tumor types: $4.4 \%$ of carcinomas (slightly more common in squamous cell carcinoma than in adenocarcinoma), $13.8 \%$ of cases of Hodgkin disease, and $7.3 \%$ of cases of non-Hodgkin lymphoma, but rare in sarcomas (3). No distinctive radiographic pattern of disease has been identified to distinguish systemic sarcoid from a sarcoidlike reaction (4). However, in addition to the lack of systemic symptoms in patients with sarcoidlike reactions, various immunohistochemical studies can be helpful, since the granulomas of sarcoid are B-cell-negative whereas those of sarcoidlike reactions are B-cell-positive (4). Also, patient demographics can be useful. Although sarcoidlike reactions have no age, sex, or racial predilection, sarcoid is most commonly encountered in young or middle-aged women, with a bias toward African-Americans (5).

Sarcoidlike reactions can occur at any time from the time of diagnosis to several years after diagnosis (4) and may occur in lymph nodes draining an area housing a malignant tumor, in the tumor itself, and even in nonregional tissues (3). Although the pathogenesis is poorly understood, one hypothesis is that granulomatous inflammation may be related to an antineoplastic immune phenomenon that represents a host defense mechanism against the spread of tumor cells (2). Sarcoidlike reactions are important to correctly diagnose because no treatment is required and they have been observed to be a good prognostic indicator (2-4).

Sarcoid is a common cause of hypermetabolic hilar and mediastinal lymph nodes on ${ }^{18} \mathrm{~F}-\mathrm{FDG}$ PET. It has been well documented that sarcoid can be imaged using ${ }^{18} \mathrm{~F}-\mathrm{FDG}$ PET, as affected tissues demonstrate a nonspecific ability to avidly accumulate ${ }^{18}$ F-FDG (2). Studies have shown that the sensitivity of ${ }^{18} \mathrm{~F}-\mathrm{FDG}$ PET/CT for identification of sites of disease involvement in sarcoid is $80 \%-100 \%$ (2). Sarcoid is a systemic inflammatory disorder of unknown cause that is characterized by the presence of noncaseating granulomas in affected tissues. The disease has multiple clinical manifestations ranging from single-organ involvement to multisystem involvement. Approximately half of patients with systemic sarcoidosis have symptoms such as erythema nodosum, uveitis, acute polyarthritis, respiratory illness, fatigue, malaise, weight loss, or fever (4). The thoracic manifestations of sarcoid, which can be seen on plain radiography and $\mathrm{CT}$, include mediastinal and hilar lymph node enlargement and pulmonary parenchymal disease. Nodal enlargement is typically bilateral and symmetric. The nodes are normally lobulated and can demonstrate 
eggshell calcification (6). Sarcoid is most commonly treated with corticosteroids.

Occupational pneumoconiosis such as silicosis can cause enlarged hilar and mediastinal nodes with variable degrees of increased uptake (7). Pneumoconiosis continues to cause more than 3,000 deaths in the United States per year, affecting workers in occupations such as surface mining despite occupational exposure regulations and prevention measures (7). The pathologic findings are secondary to excess collagen formation in the alveolar wall, which exceeds the ongoing equilibrium of collagen synthesis and degradation (7). Usually, a diffuse reticular, nodular, or reticulonodular pattern of pulmonary parenchymal disease is identified on chest radiographs or CT. The enlarged hilar and mediastinal nodes can demonstrate eggshell calcification and are often indistinguishable from those seen in sarcoid, including their ability to accumulate ${ }^{18}$ F-FDG $(6,8)$. The patient may be asymptomatic initially, although respiratory symptoms usually progress in correlation with radiographic changes (6). Pulmonary massive fibrosis often shows significantly increased uptake, perhaps related to the presence of inflammatory cells such as macrophages and fibroblasts (7). The pulmonary parenchymal changes commonly seen in pneumoconiosis have not been reported in sarcoidlike reactions and thus can serve as a useful feature for differentiating between these 2 causes of hypermetabolic hilar and mediastinal adenopathy.

A variety of infectious etiologies, most commonly granulomatous infections such as histoplasmosis and tuberculosis, can cause mediastinal lymph node enlargement with increased ${ }^{18} \mathrm{~F}$-FDG uptake $(7,8)$. These granulomatous lesions are characterized by cellular infiltrates, granuloma formation, and macrophage proliferation (9). Typically, these patients have associated pulmonary parenchymal findings on chest radiographs or CT as well as signs and symptoms of acute infection. Also, bilateral hilar and mediastinal lymph node involvement is often asymmetric (6), in distinction to sarcoid. Only a small fraction of all patients with proven histoplasmosis, tuberculosis, or other granulomatous infections may show significantly increased ${ }^{18}$ F-FDG uptake (7). It is possible that only nodes with active inflammation with abundant neutrophils or macrophages show detectable ${ }^{18}$ F-FDG uptake on scans (7). Patients with either healed or inactive processes at the time of the scan may not show

TABLE 1

Features of Hypermetabolic Hilar and Mediastinal Lymph Nodes on ${ }^{18}$ F-FDG PET

\begin{tabular}{|c|c|c|}
\hline Entity & Clinical features & CT features \\
\hline $\begin{array}{l}\text { Sarcoidlike } \\
\text { reaction }\end{array}$ & $\begin{array}{l}\text { History of prior malignancy; entity may occur at } \\
\text { time of initial cancer diagnosis to years later, } \\
\text { often with no clinical evidence of disease }\end{array}$ & $\begin{array}{l}\text { No pulmonary changes; patient classically shows } \\
\text { bilateral mild to moderately enlarged hilar and } \\
\text { mediastinal lymph nodes; enlarged or hypermetabolic } \\
\text { lymph nodes may be present elsewhere }\end{array}$ \\
\hline Sarcoid & $\begin{array}{l}\text { Bias toward young and middle-aged African- } \\
\text { American women; clinical complaints often } \\
\text { include erythema nodosum, uveitis, and acute } \\
\text { polyarthritis, with nonspecific respiratory } \\
\text { symptoms, fatigue, weight loss, and fever }\end{array}$ & $\begin{array}{l}\text { Pulmonary findings may often be absent though when } \\
\text { present show nodular pattern along lymphovascular } \\
\text { bundles; patient classically has bilateral lobulated, } \\
\text { mild to moderately enlarged hilar and mediastinal } \\
\text { lymph nodes; lymph nodes may have peripheral } \\
\text { thin egg-shell calcifications }\end{array}$ \\
\hline Pneumoconiosis & $\begin{array}{l}\text { History of occupational exposure such as surface } \\
\text { mining; patient may be asymptomatic early on } \\
\text { and then develop shortness of breath and } \\
\text { respiratory symptoms along with pulmonary } \\
\text { changes }\end{array}$ & $\begin{array}{l}\text { Diffuse reticular or nodular/reticulonodular pattern of } \\
\text { pulmonary disease; lung findings can coalesce into } \\
\text { massive pulmonary fibrosis; lymph nodes often } \\
\text { have egg-shell calcifications }\end{array}$ \\
\hline Infection & $\begin{array}{l}\text { No age or sex predilection; signs and symptoms } \\
\text { of respiratory infection are present (i.e., fever, } \\
\text { productive cough, leukocytosis, and elevated } \\
\text { C-reactive protein) }\end{array}$ & $\begin{array}{l}\text { Pulmonary features of infection (bronchopulmonary } \\
\text { nodules, infiltrate, or consolidation); lymph nodes } \\
\text { ipsilateral to pulmonary abnormality are typically } \\
\text { at upper limit of normal size to mildly enlarged }\end{array}$ \\
\hline $\begin{array}{l}\text { Radiation-induced } \\
\text { inflammation }\end{array}$ & $\begin{array}{l}\text { History of prior malignancy with treatment by } \\
\text { radiotherapy; patient may be asymptomatic or } \\
\text { have dyspnea or nonproductive cough }\end{array}$ & $\begin{array}{l}\text { Sharply marginated, localized fibrotic area of lung; } \\
\text { lymph nodes ipsilateral to fibrosis are normal to } \\
\text { mildly enlarged }\end{array}$ \\
\hline Lymphoma & $\begin{array}{l}\text { Age predilection based on type of lymphoma; } \\
\text { patient may present with weight loss, night } \\
\text { sweats, fever, decreased appetite }\end{array}$ & $\begin{array}{l}\text { Rarely has pulmonary findings; marked nodal } \\
\text { enlargement is often bilateral though may be } \\
\text { asymmetric; conglomerate nodal masses may } \\
\text { be present }\end{array}$ \\
\hline $\begin{array}{l}\text { Bronchogenic } \\
\text { carcinoma }\end{array}$ & $\begin{array}{l}\text { Older individuals, often with history of smoking or } \\
\text { pulmonary fibrosis, present with weight loss, } \\
\text { decreased appetite, hemoptysis }\end{array}$ & $\begin{array}{l}\text { Lung mass or nodule; nodal enlargement is typically } \\
\text { ipsilateral to lesion }\end{array}$ \\
\hline Metastases & $\begin{array}{l}\text { History of malignancy, possibly with increasing tumor } \\
\text { markers suggestive of disease recurrence; is most } \\
\text { commonly seen in tumors with predilection for } \\
\text { mediastinal or hilar metastases (i.e., esophageal } \\
\text { cancer or head and neck cancer) }\end{array}$ & $\begin{array}{l}\text { Lymph nodes often asymmetric or unilateral; evidence } \\
\text { of metastases may be present in lungs, bones, } \\
\text { or elsewhere }\end{array}$ \\
\hline
\end{tabular}


high ${ }^{18}$ F-FDG uptake (7). Granulomatous infectious etiologies can be readily excluded on the basis of stains and cultures for acid-fast bacilli and fungi. There have been historical reports of possible tuberculoidlike reactions in patients with underlying malignancy as well (4).

In patients with prior radiation to the mediastinum, the radiation-induced inflammatory reaction can cause increased ${ }^{18} \mathrm{~F}$-FDG uptake (7). These patients may be asymptomatic or present with dyspnea and a nonproductive cough. Radiation fibrosis can usually be differentiated from other disease entities if CT shows a sharply marginated, localized area of fibrosis that directly corresponds to the radiation port site $(6)$.

Mediastinal lymph node enlargement and increased ${ }^{18} \mathrm{~F}$ FDG uptake is common in Hodgkin disease and non-Hodgkin lymphoma. In most patients, the nodal enlargement is bilateral but asymmetric. It is not uncommon for the nodes to form conglomerate enlarged nodal masses (6). Also, as well as metastatic disease from the extrathoracic tumor in question - in this case laryngeal cancer-metastatic disease from other extrathoracic and thoracic primaries should be considered. Bronchogenic carcinoma deserves special consideration given that it is the most common cause of metastatic mediastinal disease (6). In most patients, symptoms and additional imaging findings suggest the presence of a primary tumor in the lung. Lymph node enlargement is often on the side of the cancer and when bilateral is asymmetric. Like lymphoma, metastatic disease often forms conglomerates of enlarged nodal masses (6).

In the case of patient $A$, the patient presented with hypermetabolic bilaterally symmetric hilar and mediastinal (aortopulmonary window and subcarinal) lymph nodes - some that were enlarged and others that were subcentimeter in size. No conglomerate enlarged nodal masses were appreciated, and no associated pulmonary parenchymal disease was identified. The patient had no signs or symptoms of systemic sarcoid or acute infection. Mediastinal lymph node fine-needle aspiration and core biopsy were performed, demonstrating granulomatous inflammation with no malignant cells and negative acid fast Bacillus and fungal stains. Patient B presented in a similar manner, with the addition of hypermetabolic lymph nodes in the right axilla and neck. Likewise, she had no signs or symptoms of systemic sarcoid or acute infection and multiple biopsies were performed, all demonstrating granulomatous inflammation without additional abnormality.
It is important to be aware of and consider the numerous diseases that can cause increased ${ }^{18} \mathrm{~F}-\mathrm{FDG}$ uptake in hilar and mediastinal lymph nodes and give a false-positive interpretation of metastatic mediastinal disease (Table 1). This is especially true because most diseases differ in prognosis and treatment. Although some entities such as tuberculosis and radiation fibrosis may be evident from clinical and radiologic findings, others such as sarcoidlike reaction are difficult to differentiate from metastatic disease, and tissue sampling or biopsy is often required.

\section{CONCLUSION}

Hypermetabolic hilar and mediastinal adenopathy in a patient with a known extrathoracic malignancy poses a frequent diagnostic dilemma in ${ }^{18}$ F-FDG PET. Although metastatic disease is the most worrisome etiology and a common cause, a differential diagnosis including lung cancer with nodal spread of disease, lymphoma, infection (to include tuberculosis), sarcoid, other granulomatous disease processes, and a sarcoidlike reaction to malignancy should be considered. Careful scrutiny of clinical features may help point to other possible benign diagnoses, which may avert biopsy or even chemoradiation.

\section{ACKNOWLEDGMENT}

No potential conflict of interest relevant to this article was reported.

\section{REFERENCES}

1. Gupta NC, Frank A, Dewan N. Solitary pulmonary nodules: detection of malignancy with PET with 2-[F-18]-flouro-2-deoxy-D-glucose. Radiology. 1992;184: 441-444.

2. Chowdhury FU, Sheerin F, Bradley K, Gleeson F. Sarcoid-like reaction to malignancy on whole-body integrated F18-FDG PET/CT: prevalence and disease pattern. Clin Radiol. 2009;64:675-681.

3. Brincker H. Sarcoid reactions in malignant tumours. Cancer Treat Rev. 1986; 13:147-156.

4. Hunsaker AR, Munden R, Pugatch R, Mentzer S. Sarcoidlike reaction in patients with malignancy. Radiology. 1996;200:255-261.

5. Hoang DQ, Nguyen ET. Sarcoidosis. Semin Roentgenol. 2010;45:36-42.

6. Brant W, Helms C. Fundamentals of Diagnostic Radiology. Philadelphia, PA: Lippincott Williams and Wilkins 2006:389-416.

7. Alavi A, Gupta N, Alberini J, et al. Positron emission tomography imaging in nonmalignant thoracic disorders. Semin Nucl Med. 2002;32:293-321.

8. Karam M, Roberts-Klein S, Shet N, Chang J, Feustel P. Bilateral hilar foci on ${ }^{18}$ F-FDG PET scan in patients without lung cancer: variables associated with benign and malignant etiology. J Nucl Med. 2008;49:1429-1436.

9. Goo JM, Im J, Do K, et al. Pulmonary tuberculoma evaluated by means of FDG PET: findings in 10 cases. Radiology. 2000;216:117-121. 